\title{
Article \\ Effects of Timing Noise on Square-Wave Optoelectronic Oscillators
}

\author{
David S. Citrin ${ }^{1,2}$
}

check for

updates

Citation: Citrin, D.S. Effects of Timing Noise on Square-Wave Optoelectronic Oscillators. Appl. Sci. 2021, 11, 12038. https://doi.org/ 10.3390/app112412038

Academic Editor: Wolfgang Elsaesser

Received: 3 November 2021

Accepted: 12 December 2021

Published: 17 December 2021

Publisher's Note: MDPI stays neutral with regard to jurisdictional claims in published maps and institutional affiliations.

Copyright: (c) 2021 by the authors. Licensee MDPI, Basel, Switzerland. This article is an open access article distributed under the terms and conditions of the Creative Commons Attribution (CC BY) license (https:// creativecommons.org/licenses/by/ $4.0 /)$.
1 School of Electrical and Computer Engineering, Georgia Institute of Technology, Atlanta, GA 30332-0250, USA; david.citrin@ece.gatech.edu

2 Georgia Tech-CNRS IRL2958, Georgia Tech Lorraine, 2 Rue Marconi, 57070 Metz, France

\begin{abstract}
Optoelectronic oscillators produce microwave-modulated optical beams without external modulation. The most commonly studied types produces narrow-band output, i.e., optical output modulated by a sinusoid, in which case phase noise determines key figures of merit that limit device performance. Nonetheless, other types of modulated signals have been exhibited by optoelectronic oscillators, including square waves. In this work we provide a theoretical treatment of the power spectral density of a microwave self-modulated optical periodic, but non-sinusoidal, oscillator in the presence of timing noise (as phase noise is only defined for a single sinusoid) and focus on the case of square waves. We consider the effects of timing noise on the power spectral density and autocorrelation function of the modulation signal.
\end{abstract}

Keywords: microwave photonics; optoelectronic oscillator; timing noise

\section{Introduction}

Narrow-band optoelectronic oscillators (OEO) comprise a class of optoelectronic devices that spontaneously produce a narrow-band optical signal, which is amplitudemodulated by a sinusoid of microwave frequency [1-7]. By OEO, we refer, in general, to optoelectronic-based systems that spontaneously produce microwave-modulated optical signals, i.e., without external modulation. For example, we have shown that a laser diode with time-delayed optical feedback [6] or with combined optical and optoelectronic feedback [7] can result in output of a narrow-band microwave-modulated optical signal. OEO's spontaneously producing other types of amplitude modulation [8], such as square waves (SQ) [9] and pulses trains [10], have recently been demonstrated. Phase noise is an essential figure of merit defining sinusoidal OEO performance [2,4,6,7,11-16]. More generally, we can think of phase noise as a consequence of timing noise. While there are many underlying physical mechanisms that may give rise to timing noise [12,13], one might still ask how timing noise, in a generic sense, affects device operation in the case of a non-sinusoidal (NS) OEO. Our aim in this study is to provide an analytically tractable framework for the theoretical description of timing noise in NS OEO producing periodically modulated optical output. NS OEOs may include optical and microwave frequency comb sources [17], OEOs who output signals consist of a select set of harmonics of the fundamental modulation frequency, of sources of pulse trains of specified shape. Square-wave (SW) generation by semiconductor-laser-based systems has been demonstrated, for example, in VCSELs [18-21], edge-emitters [22-27], semiconductor ring lasers (SRLs) [28,29], and quantum dot lasers [30]. In the above, SW generation is achieved without external modulation, but the square waveform results from the internal dynamics of the system. Specifically, we focus here on a SW OEO [9]. Such devices may be of interest, for example, to generate optical binary bit trains for digital communications and data processing.

Timing noise OEOs can have numerous origins [5]. For example, it could involve fluctuations in the optical delay in a feedback loop or in the laser cavity, or fluctuations in the electronics driving the system (so-called technical noise, e.g., in the injection current). 
Our approach aims to be sufficiently generic, to provide a simple and analytically tractable model for timing noise (not accounting for technical noise), though the model clearly may be simplistic and omit important noise mechanisms.

To suppress phase noise in an OEO, one approach is to inject the output of a highspectral-purity oscillator (henceforth, the reference oscillator) into an OEO, which may otherwise show considerable phase noise [31,32]. Thus, by synchronizing the OEO to the reference oscillator, the latter may stabilize the former [33-35]. This approach can result in substantial reduction in the phase noise present in the output [36-39]. This reduction is often observed as a narrowing of the power spectral density (PSD) of the oscillator output near the center of the spectral line with a line shape composed of a narrow line center superimposed on a broad pedestal. This line shape is due to the imposition of the reference oscillator of a long-time overall phase correlation on top of faster phase noise around the slowly wandering value.

There are a range of theoretical viewpoints to timing noise in oscillators. One method is to describe the noise by a physical or circuit model, or to assume a system perspective [40-43]. By the time the noise is manifested in the output, it has been processed (linearly or nonlinearly) and filtered. Other approaches employ stochastic techniques [44-46]. Stabilization by means of a reference oscillator (sometimes called injection locking) relying on oscillator synchronization has been employed for decades [47,48]. Various timing-noise studies of stabilization of electronic oscillators [43] and OEOs [31] based on the framework of Refs. [47-49] can be found in the literature.

Our model for timing noise is discussed in Refs. [17,50]. It is motivated by considering an oscillator physically producing the output signal, in our case, the NS OEO, stabilized by synchronization to a reference oscillator that is much more accurate than the NS OEO. We do not specify physically how this stabilization is achieved; it is characterized by the statistical parameters discussed below. This approach has the virtue that our theoretical treatment remains generic. The NS OEO is a considered to be a self-sustaining oscillator in the sense of Lax [45]. That is, in the absence of stabilization, its frequency fluctuates randomly about its nominal value, leading to the timing of this oscillator to wander being described by a one-dimensional random walk. For the reference oscillator we shall consider three cases specializing our results to the SW OEO: (A) it is absent; (B) it is an ideal error-free reference; and $(C)$ it is itself a self-sustaining oscillator, but with far slower diffusion than the unstabilized NS OEO. For case (B), the NS OEO timing noise generically obeys an Adler equation [47], which is conveniently treated as an Uhlenbeck-Ornstein process of one-dimensional diffusion in a harmonic potential [51]. We shall in fact see that (A) and (B) are special cases of $(\mathrm{C})$. Note that case $(\mathrm{C})$ is essentially the Maxwell-Voigt model developed to describe diffusion in viscoelastic fluids [52,53]. Certainly, other types of timing noise exist [54-57]. We do not consider the effects of time-delayed feedback, such as is present in many OEOs [31], nor do we consider filtering of the noise by either linear or nonlinear elements between the primary noise source and the timing noise acting directly on the output signal. In addition, we do not account for colored noise or multiple noise sources. Nonetheless, the model provides a physically reasonable account for an important source of timing noise, namely fluctuations in propagation time within individual elements of the OEO and the approach leads to closed-form expressions for important spectral and time-domain properties. Certainly, additional effects can be incorporated into the model.

NS OEO performance is also expected to suffer from phase noise. Because a given timing noise $\varphi(t)$ results in difference phase noise $n \omega_{0} \varphi(t)$ for the various Fourier components $n$ of an NS OEO with period $\omega_{0}^{-1}$, we use the term timing noise here instead of phase noise. In Section 2, we outline the methods to treat the problem of timing noise in NS OEOs. Taking the nominal modulation signal produced by the NS OEO to be $f(t)$, we consider the effects of $\varphi(t)$ on the autocorrelation function (acf) $\mathcal{F}(\tau)$ of $f(t)$ and on the PSD $S_{f}\left(\omega_{0}\right)$. We consider the three cases, (A)-(C) mentioned above. Section 3 presents results for a case study based on a square-wave modulated signal. In Section 4 we conclude. 


\section{Methods}

We start with a real periodic modulation signal as the modulation of the NS OEO output,

$$
f(t)=\sum_{n=1}^{\infty}\left\{a_{n} \cos \left[n \omega_{0}(t+\varphi(t))\right]+b_{n} \sin \left[n \omega_{0}(t+\varphi(t))\right]\right\}
$$

with $\omega_{0}$ the modulation frequency and $a_{n}, b_{n} \in \mathbb{R}$ the Fourier coefficients. Below, we specify the Fourier coefficients for the SW; however, the theoretical treatment in this section applies for a general NS OEO. We omit any possible dc term being of no interest. Note that $f(t)$ is the amplitude modulation, i.e., slowly varying envelope, of the optical signal and does not include the optical carrier itself. The timing noise is described by $\varphi(t)$, a random signal of zero mean, $\langle\varphi(t)\rangle=0$ with $\langle a(t)\rangle$. The time average of $a(t)$ is defined by

$$
\langle a(t)\rangle=\lim _{\hat{T} \rightarrow \infty} \frac{1}{\hat{T}} \int_{-\hat{T} / 2}^{\hat{T} / 2} d t a(t) .
$$

The PSD of $f(t)$ is

$$
S_{f}(\omega)=\lim _{\hat{T} \rightarrow \infty} \frac{1}{\hat{T}} \int_{-\hat{T} / 2}^{\hat{T} / 2} d t \int_{-\hat{T} / 2}^{\hat{T} / 2} d t^{\prime} e^{i \omega\left(t-t^{\prime}\right)} \mathcal{G}\left(t, t^{\prime}\right)
$$

where $\mathcal{G}\left(t, t^{\prime}\right)=\left\langle f(t) f\left(t^{\prime}\right)\right\rangle$. As stated above, we will consider the following cases: (A) absence of a reference oscillator; (B) presence of an ideal reference oscillator; and (C) a reference oscillator, though of nonzero bandwidth, whose phase will be permitted to wander over long timescales. In all cases, we assume the ergodic hypothesis, whereby we may freely replace time averages over $\varphi(t)$ by configuration averages over the random variable.

It will be useful to rewrite $f(t)$ as

$$
f(t)=\frac{1}{2} \sum_{n=1}^{\infty}\left\{c_{n} e^{i n \omega_{0}[t+\phi(t)]}+c_{n}^{*} e^{-i n \omega_{0}[t+i \phi(t)]}\right\}
$$

where $c_{n}=a_{n}-i b_{n}$, whence (see definition of $\mathcal{G}\left(t, t^{\prime}\right)$ and Equation (1))

$$
\begin{aligned}
\mathcal{G}\left(t, t^{\prime}\right)= & \frac{1}{4} \sum_{n, n^{\prime}=1}^{\infty}\left[c_{n} c_{n^{\prime}} e^{i \omega_{0}\left(n t+n^{\prime} t^{\prime}\right)}\left\langle e^{i \omega_{0}\left[n \varphi(t)+n^{\prime} \varphi\left(t^{\prime}\right)\right]}\right\rangle+\right.\text { c.c. } \\
& \left.+c_{n} c_{n^{\prime}}^{*} e^{i \omega_{0}\left(n t-n^{\prime} t^{\prime}\right)}\left\langle e^{i \omega_{0}\left[n \varphi(t)-n^{\prime} \varphi\left(t^{\prime}\right)\right]}\right\rangle+\text { c.c. }\right]
\end{aligned}
$$

where c.c. denotes the complex conjugate of the preceding term. Next, define the relative and average time variables $\tau$ and $T$ such that $t=T+\frac{\tau}{2}$ and $t^{\prime}=T-\frac{\tau}{2}$. To proceed,

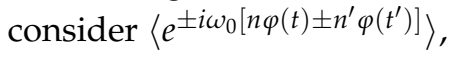

$$
\left\langle e^{ \pm i \omega_{0}\left[n \varphi(t) \pm n^{\prime} \varphi\left(t^{\prime}\right)\right]}\right\rangle=\left\langle e^{ \pm i \omega_{0}\left[n \varphi(T+\tau / 2) \pm n^{\prime} \varphi(T-\tau / 2)\right]}\right\rangle=\left\langle e^{i \omega_{0}\left[n \varphi(\tau) \pm n^{\prime} \varphi(0)\right]}\right\rangle
$$

since time averages of functions of $\varphi(t)$ only depend on the relative time since it is a stationary random variable [50]. We have

$$
\begin{aligned}
\mathcal{G}\left(t, t^{\prime}\right)= & \frac{1}{4} \sum_{n, n^{\prime}=1}^{\infty}\left\{\left[c_{n} c_{n^{\prime}} e^{i \omega_{0}\left(n+n^{\prime}\right) T} e^{i \omega_{0}\left(n-n^{\prime}\right) \tau / 2}+\text { c.c. }\right]\left\langle e^{i \omega_{0}\left[n \varphi(\tau)+n^{\prime} \varphi(0)\right]}\right\rangle\right. \\
& \left.+\left[c_{n} c_{n^{\prime}}^{*} e^{i \omega_{0}\left(n-n^{\prime}\right) T} e^{i \omega_{0}\left(n+n^{\prime}\right) \tau / 2}+\text { c.c. }\right]\left\langle e^{i \omega_{0}\left[n \varphi(\tau)-n^{\prime} \varphi(0)\right]}\right\rangle\right\} .
\end{aligned}
$$

Now consider $S_{f}(\omega)$, which we can write as

$$
S_{f}(\omega)=\lim _{\hat{T} \rightarrow \infty} \frac{1}{\hat{T}} \int_{-\infty}^{\infty} d \tau e^{i \omega \tau} \int_{-\frac{\hat{T}}{2}}^{\frac{\hat{T}}{2}} d T \mathcal{G}\left(t, t^{\prime}\right)
$$


This involves $\lim _{\hat{T} \rightarrow \infty} \hat{T}^{-1} \int_{-\hat{T} / 2}^{\hat{T} / 2} d T e^{i \omega_{0}\left(n \pm n^{\prime}\right) T}=\delta_{n, \mp n^{\prime}}$. In as much as $n, n^{\prime} \in \mathbb{Z}^{+}$, we never encounter $n=-n^{\prime}$, and so, only the terms in the second line of Equation (5) contribute to $S_{f}(\omega)$. Collecting terms containing common factors and using the stationarity of $\varphi(t)$, we have

$$
S_{f}(\omega)=\frac{1}{2} \sum_{n=1}^{\infty}\left|c_{n}\right|^{2} \int_{-\infty}^{\infty} d \tau e^{i \omega \tau} \cos n \omega_{0} \tau\left\langle e^{i n \omega_{0} \Phi(\tau)}\right\rangle
$$

with $\Phi\left(t, t^{\prime}\right)=\varphi(\tau)-\varphi(0)$. Note that the assumed stationarity implies that averages depend only on $|\tau|$. We henceforth replace $T$ in averages by 0 . It proves convenient to write Equation (6) as

$$
\begin{aligned}
S_{f}(\omega) & =\int_{-\infty}^{\infty} d \tau e^{i \omega \tau} \mathcal{F}(\tau), \\
\mathcal{F}(\tau) & =\frac{1}{2} \sum_{n=1}^{\infty}\left|c_{n}\right|^{2} \cos n \omega_{0} \tau\left\langle e^{i n \omega_{0} \Phi(\tau)}\right\rangle
\end{aligned}
$$

$\mathcal{F}(\tau)$ is the acf of $f(t)$, viz.,

$$
\mathcal{G}(\tau)=\lim _{\hat{T} \rightarrow \infty} \frac{1}{\hat{T}} \int_{-\infty}^{\infty} d T \mathcal{G}\left(t, t^{\prime}\right)
$$

We can see from Equation (6) that in the limit $\varphi(t) \rightarrow 0$,

$$
S_{f}(\omega)=\frac{\pi}{2} \sum_{n=1}^{\infty}\left|c_{n}\right|^{2}\left[\delta\left(\omega-n \omega_{0}\right)+\delta\left(\omega+n \omega_{0}\right)\right],
$$

as might not be surprising. To evaluate the integral in Equation (6) in the presence of timing noise, we need to evaluate $\left\langle\exp \left[ \pm i n \omega_{0} \Phi(\tau)\right]\right\rangle$. The average of $\Phi(\tau)$ depends only on $|\tau|$, which means $\left\langle\exp \left[i n \omega_{0} \Phi(\tau)\right]\right\rangle=\left\langle\exp \left[-i n \omega_{0} \Phi(\tau)\right]\right\rangle$. In the following, we consider cases (A)-(C). The results for $\left\langle\exp \left[i n \omega_{0} \Phi(\tau)\right]\right\rangle$ are given in Refs. [17,50]. Explicit results are given below, where we consider cases $(\mathrm{A})-(\mathrm{C})$.

(A) A reference oscillator is absent. In this case, $\varphi(t)$ obeys a one-dimensional diffusion equation. One finds [45]

$$
\left\langle\exp \left[i n \omega_{0} \Phi(\tau)\right]\right\rangle=e^{-n \omega_{0} \Lambda|\tau|}
$$

where $\Lambda$ determines the rate at which $\varphi(t)$ wanders. The integration in Equation (6) is easily carried out,

$$
S_{f}(\omega)=\frac{\pi}{2} \sum_{n=1}^{\infty}\left|c_{n}\right|^{2}\left[\frac{n \omega_{0} \Lambda / \pi}{\left(\omega-n \omega_{0}\right)^{2}+\left(n \omega_{0} \Lambda\right)^{2}}+\frac{n \omega_{0} \Lambda / \pi}{\left(\omega+n \omega_{0}\right)^{2}+\left(n \omega_{0} \Lambda\right)^{2}}\right] .
$$

The PSD line shapes centered at the modulation harmonics $n \omega_{0}$ are Lorentzians [45] with width linear in $n$. As for $\left\langle\exp \left[i n \omega_{0} \varphi(\tau)\right]\right\rangle=0$, this implies $\langle f(t)\rangle=0$, which is a consequence of the fact that the absolute timing over times $t \gg \Lambda^{-1}$ wanders uniformly over the period $\omega_{0}^{-1}$.

(B) An ideal reference oscillator resonant with $\omega_{0}$ is present. Due to synchronization with the reference oscillator, $\varphi(t)$ obeys a one-dimensional diffusion equation in a sinusoidal potential, which in the low-noise limit can be approximated by a harmonic potential. From the resulting Uhlenbeck-Ornstein model [51], one finds [17,50]

$$
\left\langle\exp \left[i n \omega_{0} \Phi(\tau)\right]\right\rangle=e^{-n^{2} \omega_{0}^{2} \sigma_{\varphi}^{2}\left(1-e^{-2|\tau| / \tilde{\tau}}\right)}
$$


where $\sigma_{\varphi}^{2}=\langle\varphi(t) \varphi(t)\rangle$ is the (time-independent) variance of $\varphi(t)$ and $\tilde{\tau}$ is the autocorrelation time of $\langle\varphi(\tau) \varphi(0)\rangle$. In this case

$$
S_{f}(\omega)=\frac{1}{2} \sum_{n=1}^{\infty}\left|c_{n}\right|^{2} \int_{-\infty}^{\infty} d \tau e^{i \omega \tau} \cos n \omega_{0} \tau e^{-n^{2} \omega_{0}^{2} \sigma_{\varphi}^{2}\left(1-e^{-2|\tau| / \tilde{\tau}}\right)} .
$$

Note that the last exponential factor in the integral causes it to diverge; however, the integral can be regularized by the following procedure. Write

$$
\begin{aligned}
e^{-n^{2} \omega_{0}^{2} \sigma_{\varphi}^{2}\left(1-e^{-2|\tau| / \tilde{\tau}}\right)} & =e^{-n^{2} \omega_{0}^{2} \sigma_{\varphi}^{2}}\left[1+\left(e^{-n^{2} \omega_{0}^{2} \sigma_{\varphi}^{2} e^{-2|\tau| / \tilde{\tau}}}-1\right)\right] \\
& =e^{-n^{2} \omega_{0}^{2} \sigma_{\varphi}^{2}}\left[1+\sum_{m=1}^{\infty} \frac{\left(n \omega_{0} \sigma_{\varphi}\right)^{2 m}}{m !} e^{-2 m|\tau| / \tilde{\tau}}\right] .
\end{aligned}
$$

Clearly, the first term gives rise to a Dirac- $\delta$ function upon performing the $\tau$ integration while the second term is integrable. The exponentials in the summation over $m$ can now be easily integrated. Equation (11) can be evaluated as

$$
\begin{aligned}
S_{f}(\omega) & =\frac{\pi}{2} \sum_{n=1}^{\infty}\left|c_{n}\right|^{2} e^{-n^{2} \omega_{0}^{2} \sigma_{\varphi}^{2}}\left[\delta\left(\omega-n \omega_{0}\right)+\delta\left(\omega+n \omega_{0}\right)\right] \\
& +\frac{\pi}{2} \sum_{n=1}^{\infty}\left|c_{n}\right|^{2} e^{-n^{2} \omega_{0}^{2} \sigma_{\varphi}^{2}} \sum_{m=1}^{\infty} \frac{\left(n \omega_{0} \sigma_{\varphi}\right)^{2 m}}{m !}\left[\frac{(2 m / \tilde{\tau}) / \pi}{\left(\omega-n \omega_{0}\right)^{2}+(2 m / \tilde{\tau})^{2}}\right. \\
& \left.+\frac{(2 m / \tilde{\tau}) / \pi}{\left(\omega+n \omega_{0}\right)^{2}+(2 m / \tilde{\tau})^{2}}\right] .
\end{aligned}
$$

Thus, $S_{f}(\omega)$ is composed of two parts: a sequence of Dirac- $\delta$ functions at modulation frequencies $n \omega_{0}$ and pedestals centered at those same frequencies. The pedestals are dominated by the $m=1$ contribution for small $n$, but additional terms render the line shape non-Lorentzian. For increasing $n$, the larger- $m$ terms play a larger rôle, causing the pedestals to merge to form a continuum.

(C) A reference oscillator resonant with $\omega_{0}$ is present. Here we assume the locking oscillator has a finite but small bandwidth that wanders over sufficiently long timescales, so that on short timescales, $\varphi(t)$ roughly tracks the temporally local value of the clock due to the reference oscillator. In this case, $\Lambda$ is associated with the reference oscillator. One finds in this case $[17,50]$

$$
\left\langle\exp \left[i n \omega_{0} \Phi(\tau)\right]\right\rangle=e^{-n \omega_{0} \Lambda|\tau|} e^{-n^{2} \omega_{0}^{2} \sigma_{\varphi}^{2}\left(1-e^{-2|\tau| / \tilde{\tau}}\right)} .
$$

This last expression is also known from the Maxwell-Voigt model of diffusion in viscoelastic fluids [52,53]. Note that (A) and (B) are special cases of (C), viz., (A) is for $\sigma_{\varphi}^{2}=0$ and (B) for $\Lambda=0$. Based on the approaches employed above,

$$
\begin{aligned}
S_{f}(\omega)= & \frac{\pi}{2} \sum_{m=0}^{\infty} \sum_{n=1}^{\infty}\left|c_{n}\right|^{2} e^{-n^{2} \omega_{0}^{2} \sigma_{\varphi}^{2}} \frac{\left(n \omega_{0} \sigma_{\varphi}\right)^{2 m}}{m !} \\
& \times\left[\frac{\left(2 m / \tilde{\tau}+n \omega_{0} \Lambda\right) / \pi}{\left(\omega-n \omega_{0}\right)^{2}+\left(2 m / \tilde{\tau}+n \omega_{0} \Lambda\right)^{2}}\right. \\
& \left.+\frac{\left(2 m / \tilde{\tau}+n \omega_{0} \Lambda\right) / \pi}{\left(\omega+n \omega_{0}\right)^{2}+\left(2 m / \tilde{\tau}+n \omega_{0} \Lambda\right)^{2}}\right] .
\end{aligned}
$$


Insofar as the reference oscillator is of narrow bandwidth, we can approximate Equation (14) as

$$
\begin{aligned}
S_{f}(\omega)= & \frac{\pi}{2} \sum_{n=1}^{\infty}\left|c_{n}\right|^{2} e^{-n^{2} \omega_{0}^{2} \sigma_{\varphi}^{2}} \\
& \times\left[\frac{n \omega_{0} \Lambda / \pi}{\left(\omega-n \omega_{0}\right)^{2}+\left(n \omega_{0} \Lambda\right)^{2}}+\frac{n \omega_{0} \Lambda / \pi}{\left(\omega+n \omega_{0}\right)^{2}+\left(n \omega_{0} \Lambda\right)^{2}}\right] \\
& +\frac{\pi}{2} \sum_{m=1}^{\infty} \sum_{n=1}^{\infty}\left|c_{n}\right|^{2} e^{-n^{2} \omega_{0}^{2} \sigma_{\varphi}^{2}} \frac{\left(n \omega_{0} \sigma_{\varphi}\right)^{2 m}}{m !}\left[\frac{(2 m / \tilde{\tau}) / \pi}{\left(\omega-n \omega_{0}\right)^{2}+(2 m / \tilde{\tau})^{2}}\right. \\
& \left.+\frac{(2 m / \tilde{\tau}) / \pi}{\left(\omega+n \omega_{0}\right)^{2}+(2 m / \tilde{\tau})^{2}}\right],
\end{aligned}
$$

that is, a sequence of Lorentzians whose width is determined by the narrow-band reference oscillator and a pedestal determined by the NS OEO itself. This result is similar to Equation (13), but with Lorentzians replacing the Dirac- $\delta$ functions.

Notice that the timing error leads to increasing the broadening with $n$ in all cases. This is because, for a given timing error, the corresponding phase error for the frequency component at $n \omega_{0}$ increases linearly with $n$. The remainder of this paper is devoted to a study of an example of the modulation PSD for cases (A)-(C). We treated $f(t)=$ $\amalg[t+\varphi(t)]$ in which $c_{n}=1$ elsewhere [17]. In the next section, we consider a square wave. Note that in the limit of a purely sinusoidal OEO, the results above approach those given in Ref. [58] for a sinusoidal OEO.

\section{Results}

In this section, we present numerical results for $S_{f}(\omega)$ for the three cases, viz., (A), (B), and $(\mathrm{C})$. We consider an even unit-amplitude square wave with $50 \%$ duty cycle, $0 \mathrm{dc}$ offset, and period $t_{0}$ for which

$$
c_{n}=\frac{4}{n \pi} \sin \frac{n \pi}{2} .
$$

For simplicity, we measure time in units of $2 \pi / \omega_{0}$ and frequency in units of $\omega_{0}$, and take $t_{0}=2 \pi \omega_{0}^{-1}$.

We consider $\mathcal{F}(\tau)$ and $S_{f}(\omega)$. First, in Figure 1a, take the unstabilized oscillator [case (A) with a substantial value of $\Lambda=0.03$ )] and in Figure $1 \mathrm{~b}$ when timing noise is absent. With increasing $\tau$, as the timing wanders, the acf peaks decrease while the troughs increase, the widths of the peaks scale with $n$ (with $\tau=n t_{0}$ ), and the acf washes out, tending to zero, as seen in Figure 1a. In Figure 1b we see the case in which timing noise is absent. The acf is a sequence of triangle functions (the acf of a rectangle function) centered at $n t_{0}$.

We now compare cases (A), (B), and (C). Figure 2 shows $\mathcal{F}(\tau)$ for (a) $\Lambda=0.005, \sigma_{\varphi}=0$ [case (A)], (b) $\Lambda=0, \sigma_{\varphi}=0.1, \tilde{\tau}=10$ [case (B)], and (c) $\Lambda=0.005, \sigma_{\varphi}=0.1, \tilde{\tau}=10$ [case $(C)$ ]. In Figure $2 a$, we assume the reference oscillator is absent, but take a very small value of $\Lambda$ for the SW OEO itself for comparison with cases (B) and (C). As noted above, the widths of the autocorrelation peaks increase, the baseline rises, and the peak heights fall off with increasing $\tau$ as more distant peaks become less correlated as the timing error eventually wanders from its initial value over the timescale $\left(\omega_{0} \Lambda\right)$. Figure $2 \mathrm{~b}$ corresponds to case (B), in which an ideal reference oscillator is present $\Lambda=0$; however, $\sigma_{\varphi}=0.1$ and $\tau=10$. For $\tau \gtrsim \tilde{\tau}, \mathcal{F}(t)$ tends to periodic (see Equation (11)). In this case, there is some smoothening of the triangular acf seen in Figure 2b. Figure 2c includes a reference oscillator that is allowed to wander, albeit slowly $(\Lambda=0.005)$, and the SW OEO itself also has timing noise $\left(\sigma_{\varphi}=0.1, \tilde{\tau}=10\right)$. Here we see a combination of features noted for cases (A) and (B), viz., an initial falling off of the acf on timescale $\tilde{\tau}$ and a tendency of the acf to wash out at larger $\tau$ ith a decrease in the peak height, an increase of the troughs, and a broadening of the peaks. 

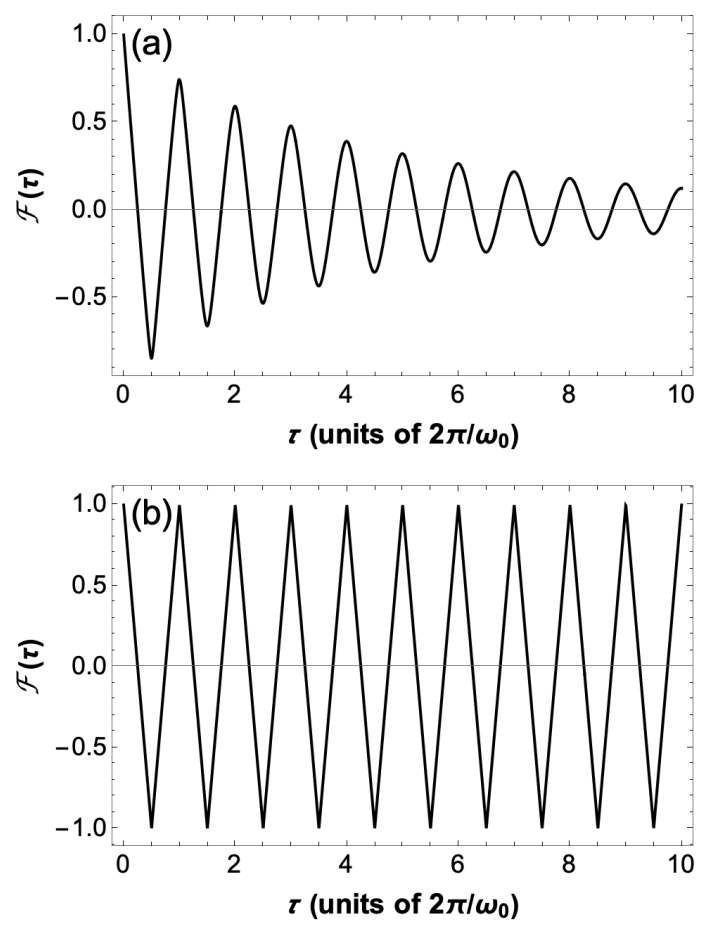

Figure 1. Autocorrelation function $\mathcal{F}(\tau)$ of the non-sinusoidal signal $f(t)$ subject to timing noise for (a) $\Lambda=0.03, \sigma_{\varphi}=0$ (representative of an unstabilized oscillator with a substantial value of $\Lambda$ ) and (b) $\Lambda=0, \sigma_{\varphi}=0$ (absence of timing noise). Time measured in units of $t_{0}=2 \pi /$ omega $_{0}$ and frequency in units of $\omega_{0}$.

We now turn to the PDF $S_{f}(\omega)$. Figure 3 shows $S_{f}(\omega)$ for (a) $\Lambda=10^{-5}, \sigma_{\varphi}=0$ [case (A), unstabilized oscillator], (b) $\Lambda=0, \sigma_{\varphi}=0.1, \tilde{\tau}=10$ [case (B), ideally stabilized oscillator], and (c) $\Lambda=10^{-5}, \sigma_{\varphi}=0.1, \tilde{\tau}=10$ [case (C), realistic stabilized oscillator]. We have chosen parameters different from those in Figure 2 to emphasize the expected features. Figure 3 a consists of a sequence of narrow Lorentzian peaks. The line width increases with $n$ and they are modulated in amplitude by $\left|c_{n}\right|^{2}$ (not visible on the plot due to the limited vertical range displayed). In Figure $3 b$, we see a sequence of peaks that, for increasing $n$, depart increasingly from Gaussian in shape (see Figure 4 below). These features provide the pedestals we shall see in Figure $3 \mathrm{c}$. Both contributions to the spectral lines are included in Figure 3c. Here, we see the pedestals seen in Figure 3b, on top of which are narrow Lorentzian peaks, as in Figure 3a. Such line shapes are known from injection-locked mode-locked laser [17].

In Figure 4, we explore the line shape in more detail. Shown is the single-sideband (SSB) spectrum of the $n=1$ line from Figure 3c. Specifically, we plot $10 \log _{10}\left[S_{f}\left(\omega_{0}+\right.\right.$ $\left.\left.\omega_{\text {offset }}\right) / S_{f}\left(\omega_{0}\right)\right]$ in $\mathrm{dBc} / \mathrm{Hz}$ where $\omega_{\text {offset }}$ is the offset frequency from the $n=1$ line center $\left(\omega=\omega_{0}\right)$. Following the peak of the Lorentzian shape near line center, we see a region corresponding to $\omega_{\text {offset }}^{-2}$ behavior-as expected for the Lorentzian—followed by a flattening due to the pedestal; of course, when this transition occurs and its details depend on the values of the parameters. 

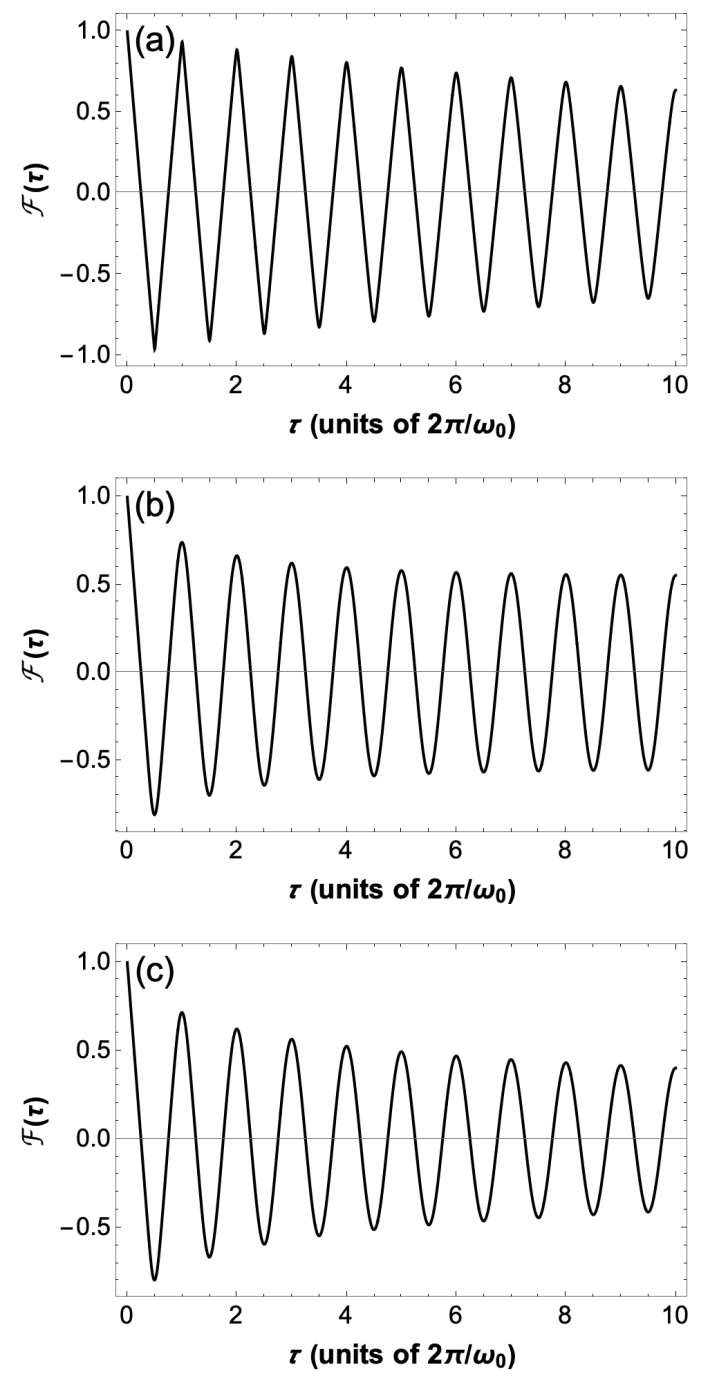

Figure 2. $\mathcal{F}(\tau)$ for $(\mathbf{a}) \Lambda=0.005, \sigma_{\varphi}=0$ (case (A), unstabilized oscillator), (b) $\Lambda=0, \sigma_{\varphi}=0.1, \tilde{\tau}=5$ [case (B), ideally stabilized oscillator], and (c) $\Lambda=0.005, \sigma_{\varphi}=0.1, \tilde{\tau}=5$ (case (C), realistic stabilized oscillator). Time measured in units of $t_{0}=2 \pi /$ omeg $a_{0}$ and frequency in units of $\omega_{0}$.

The results presented above provide a cohesive picture of the effects of timing noise on the SW OEO output in both the time and frequency domains. To recap, we account for timing noise due, for example, to fluctuations around a mean in the propagation time within an element of the OEO. This type of noise will produce fluctuations in the frequencies of the resonant optical modes of that optical element; in turn, in the absence of stabilization, the timing will wander away over time. This is the language of self-sustained oscillators in the sense of Ref. [45]. If, however, the OEO is injection locked by a second high spectral purity oscillator, the stabilization effect acts to maintain the timing around the standard imposed by this locking oscillator. This is described in terms of an Uhlenbeck-Ornstein process. However, even such a locking oscillator is also, in the end, self-sustained and, thus, may wander. We accounted for both effects using a Maxwell-Voigt model $[52,53]$ that combines aspects of the self-sustained oscillator with that of the locked oscillator. 

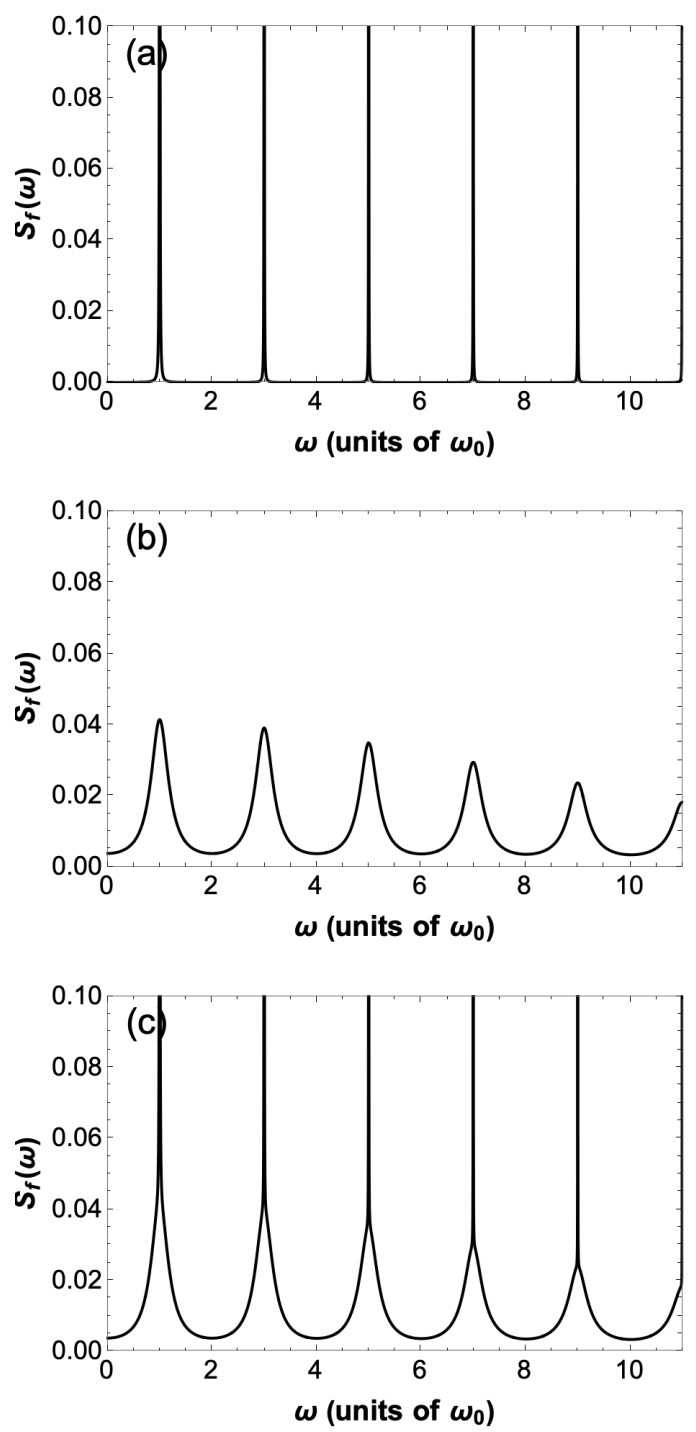

Figure 3. $S_{f}(\omega)$ for (a) $\Lambda=10^{-5}, \sigma_{\varphi}=0$ (case (A), unstabilized oscillator), (b) $\Lambda=0, \sigma_{\varphi}=0.1$, $\tilde{\tau}=10$ [case (B), ideally stabilized oscillator], and (c) $\Lambda=10^{-5}, \sigma_{\varphi}=0.1, \tilde{\tau}=10$ (case (C), realistic stabilized oscillator). Time measured in units of $t_{0}=2 \pi /$ omeg $a_{0}$ and frequency in units of $\omega_{0}$.

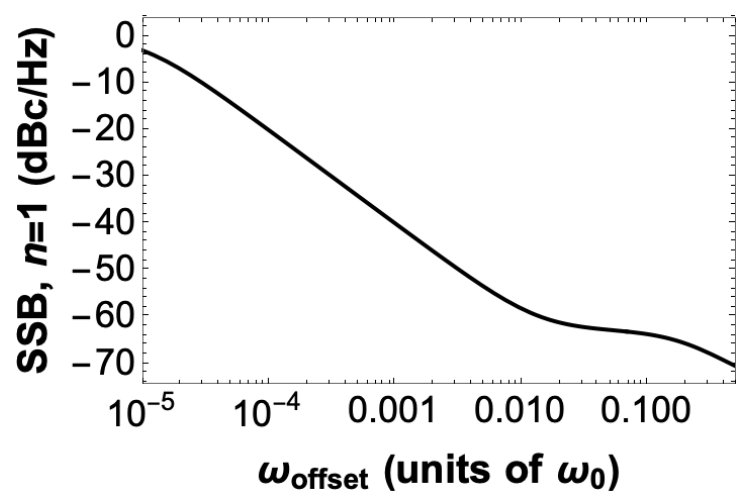

Figure 4. Single-sideband spectrum spectrum at $n=1$ peak for $\Lambda=10^{-5}, \sigma_{\varphi}=0.1, \tilde{\tau}=10$ (case (C), realistic stabilized oscillator) as in Figure 3.

\section{Conclusions}

To conclude, we presented an analytically tractable model for a NS OEO, considering three cases: (A) a reference oscillator is absent, in which case the NS OEO is a self-sustaining 
oscillator; (B) an ideal reference oscillator is present, and the NS OEO is synchronized to the reference oscillator, maintaining the timing, to be close to the absolute time standard; and (C) a reference oscillator is present that has finite bandwidth, and the NS OEO is synchronized to the reference oscillator, though the timing is not locked to the absolute time.

We assume a single primary noise source, and do not account for complete filtering or nonlinear distortion of the noise in leading to $\varphi(t)$. Time-delayed feedback is not taken into account, though many OEOs rely on feedback. This might lead to temporal correlations in $\varphi(t)$ on the timescale of the feedback time neglected here [31]. The key results are those presented in Figures 3 and 4. For case (A), the PSD is composed of a sequence of spectral lines centered at frequencies $n \omega_{0}$ of Lorentzian shape. The line widths scale with $n$ since the phase noise associated with the spectral component $n$ is $n \varphi(t)$. At the same time, the maxima decrease in height is $n^{-1}$. Thus, as $n \rightarrow \infty$, the PSD washes out. For case (B), the spectral line shape is more complex (non-Lorentzian); as $n$ increases, the line widths increase and maxima decrease in height, and eventually merge to form a continuum. For case (C), the most general case, the line shape is composed of a narrow Lorentzian peak whose widths are associated with the reference oscillator atop a broad pedestal associated with the SW OEO itself. Figure 4 provides an example of the line shape for case $(C)$ in the form of the SSB spectrum. Here, we see the $\omega_{0}^{-2}$ dependence at a small frequency offset due to the Lorentzian component and a more complex dependence at larger $\omega_{\text {offset }}$.

What we wish to emphasize is the following. In the PSD, the various Fourier peaks associated with the discrete frequency components of the periodic signal are broadened with two distinct contributions. First, there is a narrow central feature that is Lorentzian broadened due to the wandering of the stabilizing oscillator. Presumably, this broadening is not severe since we assume we have chosen a high spectral-purity oscillator specifically for this purpose. This narrow central line lies on top of a broad pedestal whose shape is dominated by the fluctuations inherent in the OEO, rather than in the stabilizing oscillator. The shape of the pedestal is complex. As the frequency of the Fourier component increases, so does the broadening. That is because, for a given timing noise $\varphi(t)$, the phase noise associated with $\varphi(t)$ increases linearly with frequency. Thus, the pedestals ultimately merge at a high frequency to form a continuum.

This study provides basic analytic results for the stabilized OEO. The model is illustrated for parameters selected to highlight the effects, and is not motivated by any experimental results; however, the treatment is general under the assumptions of the model, and can be applied not only to SW OEOs, but also to any other example of modulation that is non-sinusoidal.

Funding: We gratefully acknowledge the support from Conseil Régional Grand Est and CPER SusChemProc.

Conflicts of Interest: The author declares no conflict of interest.

\section{References}

1. Yao, X.S.; Maleki, L. High frequency optical subcarrier generator. Electron. Lett. 1994, 30, 1525-1526. [CrossRef]

2. Yao, X.S.; Maleki, L. Optoelectronic microwave oscillator. J. Opt. Soc. Am. B 1996, 13, 1725-1735. [CrossRef]

3. Poinsot, S.; Porte, H.; Goedgebuer, J.-P.; Rhodes, W.T.; Boussert, B. Continuous radio-frequency tuning of an optoelectronic oscillator with dispersive feedback. Opt. Lett. 2002, 27, 1300-1302. [CrossRef] [PubMed]

4. Maleki, L. Sources: The optoelectronic oscillator. Nat. Photonics 2011, 5, 728-730. [CrossRef]

5. Chembo, Y.K.; Brunner, D.; Jacquot, M.; Larger, L. Optoelectronic oscillators with time-delayed feedback. Rev. Mod. Phys. 2019, 91, 035006. [CrossRef]

6. Chang, C.-Y.; Wishon, M.J.; Choi, D.; Dong, J.; Merghem, K.; Ramdane, A.; Lelarge, F.; Martinez, A.; Locquet, A.; Citrin, D.S. Tunable X-Band Optoelectronic Oscillators Based on External-Cavity Semiconductor Lasers. IEEE J. Quantum Electron. 2017, 53, 2000406. [CrossRef]

7. Wishon, M.J.; Choi, D.; Niebur, T.; Webster, N.; Chembo, Y.K.; Viktorov, E.A.; Citrin, D.S.; Locquet, A. Low-Noise X-Band Tunable Microwave Generator Based on a Semiconductor Laser With Feedback. IEEE Photonics Technol. Lett. 2018, 30, 1597-1600. [CrossRef] 
8. Li, W.; Kong, F.; Yao, J. Arbitrary Microwave Waveform Generation Based on a Tunable Optoelectronic Oscillator. J. Lightwave Technol. 2013, 31, 3780-3786. [CrossRef]

9. Islam, M.S.; Kovalev, A.V.; Viktorov, E.A.; Locquet, A.; Citrin, D.S. Optical square-wave generation in a semiconductor laser with optoelectronic feedback. Opt. Lett. 2021, 46, 6031-6034. [CrossRef]

10. Islam, M.S.; Kovalev, A.V.; Viktorov, E.A.; Citrin, D.S.; Locquet, A. Microwave Frequency Comb Generation by Gain-Switching Versus Relaxation Oscillations. IEEE Photonics Technol. Lett. 2021, 33, 491-494. [CrossRef]

11. Logan, R.T.; Maleki, L.; Shadaram, M. Stabilization of oscillator phase using a fiber-optic delay-line. In Proceedings of the Proceedings of the 45th Annual Symposium on Frequency Control 1991, Los Angeles, CA, USA, 29-31 May 1991; pp. 508-512.

12. Devgan, P. A review of optoelectronic oscillators for high speed signal processing applications. ISRN Electron. 2013, $2013,401969$. [CrossRef]

13. Römisch, S.; Kitching, J.; Ferrè-Pikal, E.; Hollberg, L.; Walls, F.L. Performance evaluation of an optoelectronic oscillator. IEEE Trans. Ultrason. Ferroelectr. Freq. Control 2000, 47, 1159-1165. [CrossRef] [PubMed]

14. Hong, J.; Yao, S.X.; Li, Z.; Fang, X.Y.; Guo, J. The effect of flicker noise on the phase noise of opto-electronic oscillator. Optik 2014, 125, 1572-1574. [CrossRef]

15. Zou, X.; Liu, X.; Li, W.; Li, P.; Pan, W.; Yan, L.; Shao, L. Optoelectronic oscillators (OEOs) to sensing, measurement, and detection. IEEE J. Quantum Electron. 2016, 52, 0601116. [CrossRef]

16. Chan, S.-C.; Liu, J.-M. Tunable narrow-linewidth photonic microwave generation using semiconductor laser dynamics. IEEE J. Sel. Top. Quantum Electron. 2004, 10, 1025-1032. [CrossRef]

17. Citrin, D.S. Connection between Optical Frequency Combs and Microwave Frequency Combs Produced by Active-Mode-Locked Lasers Subject to Timing Jitter. Phys. Rev. Appl. 2021, 16, 014004. [CrossRef]

18. Sciamanna, M.; Rogister, F.; Deparis, O.; Mégret, P.; Blondel, M.; Erneux, T. Bifurcation to polarization self-modulation in vertical-cavity surface-emitting lasers. Opt. Lett. 2002, 27, 261-263. [CrossRef] [PubMed]

19. Mulet, J.; Giudici, M.; Javaloyes, J.; Balle, S. Square-wave switching by crossed-polarization gain modulation in vertical-cavity semiconductor lasers. Phys. Rev. A 2007, 76, 043801. [CrossRef]

20. Sukow, D.; Gilfillan, T.; Pope, B.; Torre, M.; Gavrielides, A.; Masoller, C. Square-wave switching in vertical-cavity surface-emitting lasers with polarization-rotated optical feedback: Experiments and simulations. Phys. Rev. A 2012, 86, 033818. [CrossRef]

21. Marconi, M.; Javaloyes, J.; Barland, S.; Giudici, M.; Balle, S. Robust square-wave polarization switching in vertical-cavity surface-emitting lasers. Phys. Rev. A 2013, 87, 013827. [CrossRef]

22. Gavrielides, A.; Erneux, T.; Sukow, D.W.; Burner, G.; McLachlan, T.; Miller, J.; Amonette, J. Square-wave self-modulation in diode lasers with polarization-rotated optical feedback. Opt. Lett. 2006, 31, 2006-2008. [CrossRef]

23. Friart, G.; Weicker, L.; Danckaert, J.; Erneux, T. Relaxation and square-wave oscillations in a semiconductor laser with polarization rotated optical feedback. Opt. Express 2014, 22, 6905-6918. [CrossRef] [PubMed]

24. Friart, G.; Verschaffelt, G.; Danckaert, J.; Erneux, T. All-optical controlled switching between time-periodic square waves in diode lasers with delayed feedback. Opt. Lett. 2014, 39, 6098-6101. [CrossRef]

25. Masoller, C.; Sukow, D.; Gavrielides, A.; Sciamanna, M. Bifurcation to square-wave switching in orthogonally delay-coupled semiconductor lasers: Theory and experiment. Phys. Rev. A 2011, 84, 023838. [CrossRef]

26. Sciamanna, M.; Virte, M.; Masoller, C.; Gavrielides, A. Hopf bifurcation to square-wave switching in mutually coupled semiconductor lasers. Phys. Rev. E 2021, 86, 016218. [CrossRef]

27. Masoller, C.; Sciamanna, M.; Gavrielides, A. Two-parameter study of square-wave switching dynamics in orthogonally delaycoupled semiconductor lasers. Philos. Trans. R. Soc. A 2013, 371, 20120471. [CrossRef]

28. Mashal, L.; Van der Sande, G.; Gelens, L.; Danckaert, J.; Verschaffelt, G. Square-wave oscillations in semiconductor ring lasers with delayed optical feedback. Opt. Express 2012, 20, 22503-22516. [CrossRef]

29. Li, S.-S.; Li, X.-Z.; Zhuang, J.-P.; Mezosi, G.; Sorel, M.; Chan, S.-C. Square-wave oscillations in a semiconductor ring laser subject to counter-directional delayed mutual feedback. Opt. Lett. 2016, 41, 812-815. [CrossRef]

30. Dillane, M.; Tykalewicz, B.; Goulding, D.; Garbin, B.; Barland, S.; Kelleher, B. Square wave excitability in quantum dot lasers under optical injection. Opt. Lett. 2019, 44, 347-350. [CrossRef]

31. Talla, A.F.; Martinenghi, R.; Goune Chengui, G.R.; Talla Mbé, J.H.; Saleh, K.; Coillet, A.; Lin, G.; Woufo, P.; Chembo, Y.K. Analysis of phase-locking in narrow-band optoelectronic oscillators with intermediate frequency. IEEE J. Quantum Electron. 2015, 51, 5000108. [CrossRef]

32. Zhou, W.; Blasche, G. Injection-locked dual opto-electronic oscillator with ultra-low phase noise and ultra-low spurious level. IEEE Trans. Microw. Theory Tech. 2005, 53, 929-933. [CrossRef]

33. Chang, H.C.; Cao, X.; Vaughan, M.J.; Mishra, U.K.; York, R.A. Phase Noise in Externally Injection-Locked Oscillator Arrays. IEEE Trans. Microw. Theory Tech. 1997, 45, 2035-2042. [CrossRef]

34. Pan, S.; Tang, Z.; Zhu, D.; Ben, D.; Yao, D.J. Injection-locked fiber laser for tunable millimeter-wave generation. Opt. Lett. 2011, 36, 4722-4724. [CrossRef]

35. Razavi, B. A study of injection locking and pulling in oscillators. IEEE J. Solid-State Circuits 2004, 39, 1415-1424. [CrossRef]

36. Banerjee, A.; Agular Dantas de Britto, L.; Mendes Pacheco, G. A theoretical and experimental study of injection-pulling for IL-PLL T optoelectronic oscillator under RF signal injection. Optik 2020, 203, 164059. [CrossRef] 
37. Menyuk, C.R.; Levy, E.C.; Okusaga, O.; Horowitz, M.; Carter, G.M.; Zhou, W. An analytical model of the dual-injection-locked opto-electronic oscillator (DIL-OEO). In Proceedings of the 2009 IEEE International Frequency Control Symposium Joint with the 22nd European Frequency and Time forum, Besancon, France, 20-24 April 2009; pp. 870-874. [CrossRef]

38. Li, C.J.; Hsiao, C.H.; Wang, F.K.; Horng, T.S.; Peng, K.C. A Rigorous Analysis of a Phase-Locked Oscillator Under Injection. IEEE Trans. Microw. Theory Tech. 2010, 58, 1391-1400.

39. Bhansali, P.; Roychowdhury, J. Gen-Adler: The generalized Adler's equation for injection locking analysis in oscillators. In Proceedings of the 2009 Asia and South Pacific Design Automation Conference, Yokohama, Japan, 19-22 January 2009; pp. 522-527. [CrossRef]

40. Hajimiri, A.; Lee, T. A State-Space Approach to Phase Noise in Oscillators; Technical Memorandum; Lucent Technologies: Murray Hill, NJ, USA, 1997.

41. Hajimiri, A.; Lee, T. A general theory of phase noise in electrical oscillators. IEEE J. Solid-State Circuits 1998, 33, 179-194. [CrossRef]

42. Schumakher, E.; Eisenstein, G. On the Noise Properties of Injection-Locked Oscillators. IEEE Trans. Microw. Theory Tech. 2004, 52, 1523-1537. [CrossRef]

43. Kalia, S.; Elbadry, M.; Sadhu, B.; Patnaik, S.; Qiu, J.; Harjani, R. A simple, unified phase noise model for injection-locked oscillators. In Proceedings of the 2011 IEEE Radio Frequency Integrated Circuits Symposium, Baltimore, MD, USA, 5-7 June 2011; pp. 1-4. [CrossRef]

44. Demir, A.; Mehrotra, A.; Roychowdhury, J. Phase noise in oscillators: A unifying theory and numerical methods for characterization. IEEE Trans. Circuits Syst. I Fundam. Theory Appl. 2000, 47, 655-674. [CrossRef]

45. Lax, M. Classical noise. V. Noise in self-sustained oscillators. Phys. Rev. 1967, 160, 290-307. [CrossRef]

46. Kärtner, F.K. Analysis of white and $f^{-\alpha}$ noise in oscillators. Int. J. Circuit Theory Appl. 1990, 18, 485-519. [CrossRef]

47. Adler, R. A study of locking phenomena in oscillators. Proc. IRE 1946, 43, 351-357. [CrossRef]

48. Paciorek, L.J. Injection locking of oscillators. Proc. IEEE 1965, 11, 1723-1728. [CrossRef]

49. Chembo, Y.K.; Volyanskiy, K.; Larger, L.; Rubiola, E.; Colet, P. Determination of phase-noise spectra in optoelectronic microwave oscillators: A Langevin approach. IEEE J. Quantum Electron. 2009, 45, 178-186. [CrossRef]

50. Citrin, D.S. Photonic Sampling Analog-to-Digital Conversion With Read-In Timing Jitter. IEEE Trans. Commun. 2021. [CrossRef]

51. Uhlenbeck, G.E.; Ornstein, L.S. On the theory of Brownian motion. Phys. Rev. 1930, 36, 823-841. [CrossRef]

52. Ferry, J.D. Viscoelastic Properties of Polymers; Wiley: New York, NY, USA, 1970.

53. Khan, M.; Mason, T.G. Random walks of colloidal probes in viscoelastic materials. Phys. Rev. E 2014, 89, 042309. [CrossRef] [PubMed]

54. Allan, D.W. Clock characterization tutorial. In Proceedings of the 15th Annual Precise Time and Time Interval Systems and Applications Meeting, Washington, DC, USA, 6-8 December 1983.

55. Sullivan, D.B.; Allan, D.W.; Howe, D.A.; Walls, F.L. Characterization of Clocks and Oscillators; NIST Technical Note 1337; National Institutes of Standards and Technology: Boulder, CO, USA, 1990.

56. Leeson, D.B.; Oscillator phase noise: A 50-year review. IEEE Trans. Ultrason. Ferroelectr. Freq. Control 2016, 63, 1208-1225. [CrossRef]

57. Lee, T.H.; Hajimiri, A. Oscillator phase noise: A tutorial. IEEE J. Solid-State Circuits 2000, 35, 326-336. [CrossRef]

58. Citrin, D.S. Power Spectral Density of Injection-Locked Optoelectronic Oscillators: Effects of Phase Noise. J. Lightwave Technol. 2021, in press. [CrossRef] 